\title{
Prevalence of oral diseases in pediatric population in Karachi, Pakistan-a cross-sectional survey
}

\begin{abstract}
Objective: The aim of the study was to create a profile of oral diseases in a paediatric Pakistani population and to compare it to available data.

Study design: This descriptive cross sectional multi-center study was designed to be conducted in the Oral and Maxillofacial Surgery clinics of tertiary care facilities. The time period of data collection was 2 years and 6 months. All pediatric patients aged 0-17 years after clinical assessment and initial diagnosis were included in the study. Study, making sample size of 1062 patients.. The data was categorized as 13 items with help of SPSS 17 and frequency of diseases according to age and gender were calculated on that basis.
\end{abstract}

Results: The result showed male to female ratio of 1:1.4. The highest numbers of cases reported were under the age of 1 year (15\%). The commonest reason for presentation was Dental Caries (44.7\%), followed by trauma, hereditary disorders, pre-cancerous lesions, others, salivary gland disorders, cysts, neoplasia, infections, inflammatory lesions, referred cases, TMJ disorders and systemic diseases.

Conclusion: Results from this study will form part of a larger nation-wide data collection study, which will form the basis to propose a strategy to establish how this study can benefit the provision of Paediatric OMF Surgery services across all regions for improved child care services.

Keywords: oral diseases, pediatric oral maxillofacial surgery, tertiary health care
Volume 6 Issue I - 2017

\author{
Sanaa Ahmed,' Syed Mahmood Haider, ${ }^{2}$ \\ Samra Bokhari ${ }^{3}$ \\ 'Sindh Institute of Oral Health Sciences, Jinnah Sindh Medical \\ University, Pakistan \\ 2Departments of Oral and Maxillofacial Surgery,Abbasi Shaheed \\ Hospital and the Karachi Medical \& Dental College, Pakistan \\ ${ }^{3}$ National Institute of Child Health Karachi, Pakistan
}

Correspondence: Sanaa Ahmed, Sindh Institute of Oral Health Sciences, Rafiqui HJ Road, Karachi, Pakistan,

Email sanaaumair@gmail.com

Received: December 08, 2016 | Published: January 23, 2017

\section{Introduction}

Oral diseases remain highly prevalent in 2010 affecting 3.9 billion people. ${ }^{1}$ Untreated caries in permanent teeth was the most prevalent condition evaluated for the entire GBD [Global Burden of Disease] 2010 Study with a global prevalence of $35 \%$ for all ages combined. ${ }^{2}$ WHO report found that oral cancer and caries increased markedly by an average of $45.6 \%$ from 1990 to 2010 in parallel with the major noncommunicable diseases like diabetes by $69.0 \% .{ }^{3}$ Pediatric Oral and maxillofacial surgery is an area of special interest within dentistry as the presentation and management of oral and maxillofacial disorder's in children differ from adults in nature and severity.4-6 According to a report, oro-dental trauma prevalence was $5-12 \%$ in children aged 6-12 years in Middle East on contrary a study conducted in Kuwait in which dental caries was the most common problem in children aged 12-16 years with males having higher DMFT score. ${ }^{7}$ Research done in Brazilian nursery going children found that caries; specifically rampant caries was more significant in low income families with little access to dental services. ${ }^{8}$ In industrialized countries, caries incidence was between $16-40 \%$ among 6 year old children and $4-33 \%$ among 12 14 year old age group. ${ }^{9}$ Several studies found that developing countries had dental infectious diseases such as dental caries as the leading cause for dental visit in children contrary to the developed countries where it was congenital diseases and oro-dental traumatic injuries..$^{2-16}$ Dental anomalies found in Kuwaiti children were missing mandibular first molar followed by mandibular second pre-molar and maxillary first molar while in Jordan maxillary hypodontia [106 patients] was prevalent than mandibular hypodontia (46 patients). ${ }^{7-17}$ Reason for missing teeth was found to be tooth loss due to caries in contrast to congenital absence. ${ }^{1-17}$ Tooth loss was the main morbidity associated with increased caries incidence and lack of dental services in underdeveloping countries. In a systemic review authors reported that prevalence of tooth loss is equivalent in both sexes and increased with increase in age. Though there is significant overall decline between 1990-2010 in its incidence and prevalence. ${ }^{16}$ In Nigeria retrospective analysis of 156 patients younger than 18 years reporting to pediatric emergency department concluded that males are mostly affected. Mean age was 13.5 year with road traffic accident as the main reason. Mandibular fractures were the most common diagnosis. ${ }^{18}$ Similar findings were observed by Bede SY. With male gender predominant. Soft tissue injuries were high in younger patients and facial fractures increased with age. Fall and puncture wounds were high in younger children and shifted towards road traffic accidents, violence, bicycle, missile and industrial injuries with increase in age. ${ }^{19}$ While Al-Shetawi quoted assault as the main reason. ${ }^{20}$ Developmental disorders in Asians are reported to be very high in comparison to others ${ }^{2}$ Specially in India and Pakistan ratio of consanguineous marriages are very high causing a lot of genetic disorders to pass from one generation to others. According to a study conducted in northern Pakistan, 117 cases of cleft lip/palate out of 61, 156 live births are reported. Out of those 117 cases, 18 had family history of cleft. 11 cases of cleft palate have syndromes associated with them. ${ }^{21}$ Improvements in population oral health may imply substantial economic benefits not only in terms of reduced treatment costs but also because of fewer productivity losses in the labor market. ${ }^{13}$ Although global improvement in oral health was attributed to oral health research, no study was found focusing solely on pediatric oral health. Current studies on pediatric patients discuss dento-facial trauma and oral pathology separately. ${ }^{7-20}$ No research 
study has been done which gives a single profile of pediatric oral diseases inclusive of oral-facial trauma.

\section{Materials and methods}

A multi-center prospective cross-sectional survey was conducted in the dental clinics of four tertiary care hospitals [Karachi Medical and Dental College, Abbasi Shaheed Hospital, the National Institute of Child Health and the National Institute of Oral Diseases] in Karachi, Pakistan. Data was collected from June 2012-December 2014 after approval from Institutional Review Board of Karachi Medical and Dental College [[Ref No ERC- 41/12]. The study objectives were explained to parents by dentist and written informed consent from parents was taken for participation in study. The sample size was calculated using previous study which found the prevalence of oral diseases at 3.9 billion (54.45\%) of global population] $]^{1,2}$ The margin of error was $5 \%$ and confidence level of $99 \%$, we would need a sample size of 664 for the population of 20 million people at the response distribution of $50 \%$. We collected a sample of 1,000 patients.

Inclusion criteria: All the dental patients aged $0 \geq 17$ years recruited from dental clinics of tertiary care hospitals. Permission from local ethical review board was taken as a part of the protocol [Ref No ERC41/12].

Exclusion criteria: All patients with life-threatening systemic diseases were excluded from the study.

Study procedures: Study procedures included the completion of a questionnaire [by the participants' parents/guardians], an oral mucosal examination, a dental examination, and the assessment of gingival and periodontal health.

Performa data: A standardized performa was designed to collect information on socio-demographic characteristics, oral health status, oral diagnosis, and treatment. All the diseases pertaining to Oral and Maxillofacial region were divided into 13 categories with few categories further divided into sub-categories.

\section{Statistical analysis}

A descriptive statistical analysis of variables was performed using SPSS-21. Various demographic, qualitative and quantitative variables were represented using pie-charts and various graphs.

\section{Results}

Altogether 1062 pediatric patients were included in the study from all the centers, consisting of 617 males and 445 females (Figure 1) (Figure 2). or the age related frequency of cases (Figure 3) shows that over $15 \%$ of the cases were under 1 year of age followed by children between the ages of $11-12$ years $(8.1 \%), 9-10$ years of age $(7.7 \%)$, $6-7$ years of age $(7.6 \%), 5-6$ years of age $(6.4 \%), 7-8$ years of age $(6.03 \%), 3-4$ tears of age $(5.3 \%), 8-9$ years of age $(5.2 \%), 2-3$ years of age $(4.8 \%), 15-16$ years of age $(4.8 \%), 4-5$ years of age $(4.7 \%), 14-15$ years of age $(4.45 \%), 10-11$ years of age $(4.35 \%), 1-2$ years of age $(3.65 \%), 12-13$ years of age $(3.06 \%)$ and $13-14$ years of age $(2.86 \%)$ respectively. Analysis of cases on the basis of categories (Figure 4) showed that Dental problems $(44.7 \%)$ were the most common reason behind reporting followed by Trauma [18.36\%], Hereditary problems [15.25\%], Precancerous lesions (6.49\%), Salivary gland disorders (3.38\%), TMJ disorders (2.7\%), Others $(2.2 \%)$, Cysts $(2.16 \%)$, Neoplasia (2.16\%), Infections ( $0.7 \%)$, Inflammatory lesions $(0.37 \%)$, Referred cases $(0.09 \%)$ and Systemic diseases $(0 \%)$. Amongst the dental problems the most common issues were caries (97\%) followed by dental anomalies (less than $1 \%$ ) and un-erupted or impacted teeth(less than $1 \%$ ) (Figure 5). Dental anomalies included neonatal teeth and supernumerary and supplemental teeth (16 in number), canine was the frequently impacted teeth reported in 3 cases. Out of 1062 cases 195 contributed to trauma. Soft tissue problem contributed to $49 \%$ of the cases with lacerations being the commonest. Second common being the mandible fractures (29\%), maxilla fractures $(13 \%)$ and Dento-alveolar fractures the least common contributing only $9 \%$ of injuries. The most common site of injury was body of the mandible (24\%) followed by multiple fractures of mandible (24\%), condyler $(22 \%)$, Parasymphysis (16\%), Angle (7.27\%) and intracapsular $(1.81 \%)$. Most reported cases were between the age group 10-11 years with most common reason of injury as road traffic accident. Frequency of common disease in Hereditary/congenital category were mostly cleft lip/palate $(98.1 \%$ ) cases followed by fibrous dysplasia (less than $1 \%$ ), encephalocoele (less than $1 \%$ ) and craniosyntosis (less than 1\%). Among the Cystic lesions, Dentigerous was the most common comprising of 16 cases (69.6 percent) followed by Radicular (3 cases), Dermoid, OKC, Sebaceous Cyst ( 2 cases each, 8.7 percent) and Epidermoid ( 1 case 4.3 percent). Mucocoeles were most common salivary gland problem having 32 (88.9 percent) out of 36 cases and ranula having 4 cases (11.1 percent). Among the Pre-cancerous category Oral Submusous Fibrosis contributed to 68 cases out of 69 and erythroplakia having 1 case only while Neoplastic lesions category showed Squamous cell papilloma 7 cases, Hemangioma 6 cases, Ameloblastoma 4 cases, followed by Lipoma, Fibroma having 2 cases each and Neurofibroma and polyp having 1 case each. Joint disorders had 29 patients categorized under Temporomandibular ankyloses with 15 cases followed by TMPDS 6 cases, dislocation 5 cases, Arthritis having 2 cases and Degenerative disease of the joint having 1 case. In category of-others most common was Tongue tie/HighFrenum (23 cases) followed by Bullet removal (4 cases), Pyogenic Granuloma (4 cases), Facial palsy (3 cases), Gingival Hyperplasia (2 cases), Trigeminal Neuralgia (1 case), Double lip (1 case) and Muscular Hypertrophy ( 1 case). In the last category of Referral, only 1 case was referred to ENT for evaluation of ear infection mimicking tooth pain.

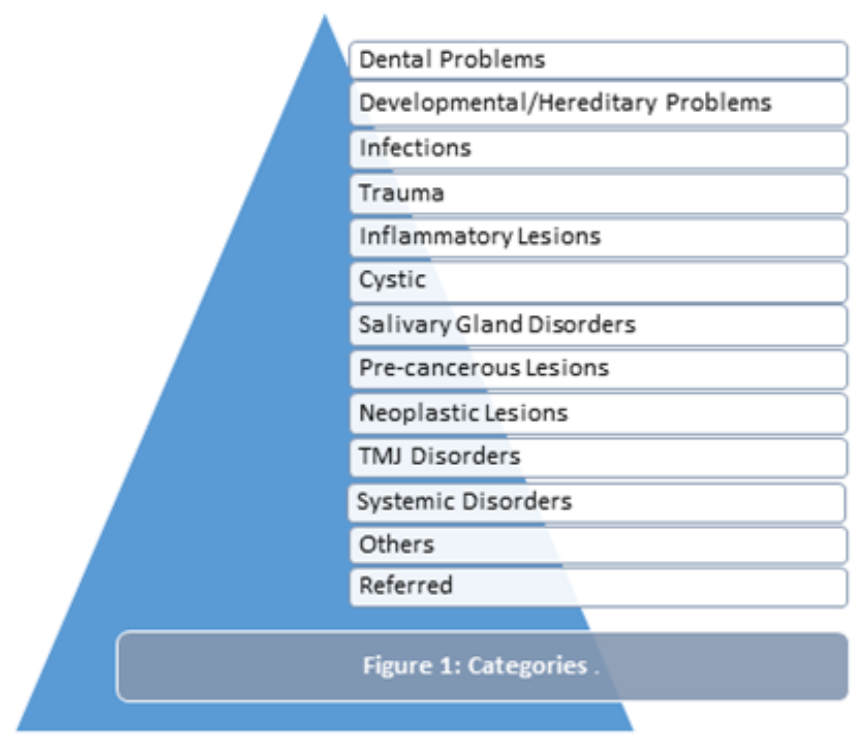

Figure I Categories. 


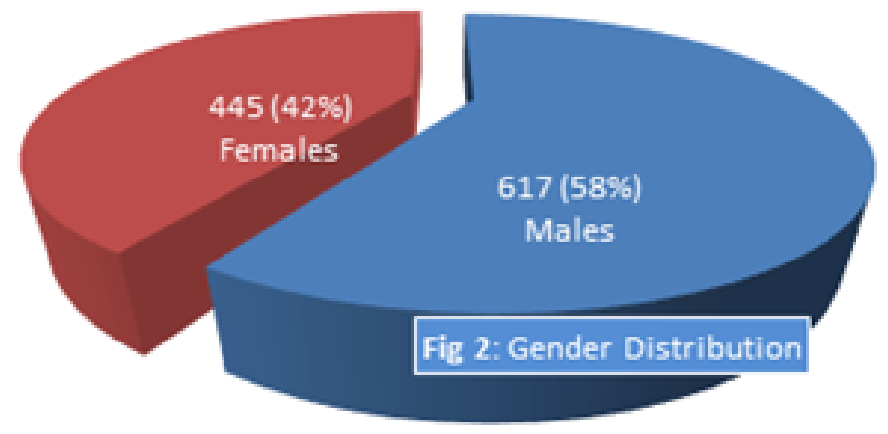

Figure 2 Gender distribution.

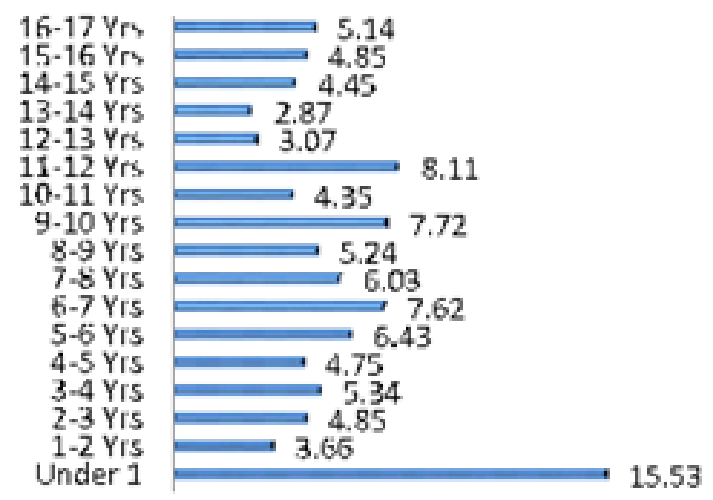

Fig 3: Frequency of Cases According to Age (in \%)

Figure 3 Frequency of cases according to age.

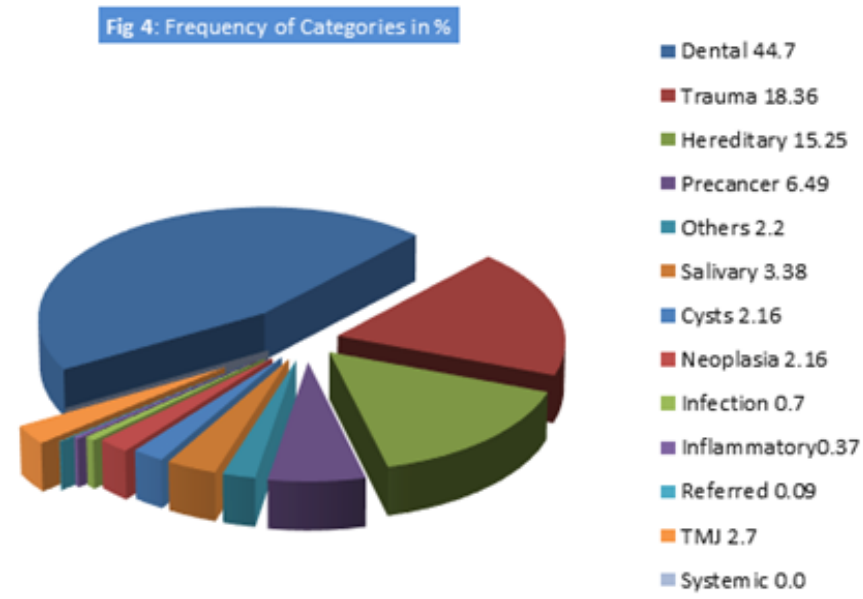

Figure 4 Frequency of categories in \%.

\section{Fig 5: Frequency of Dental Problems in $\%$}

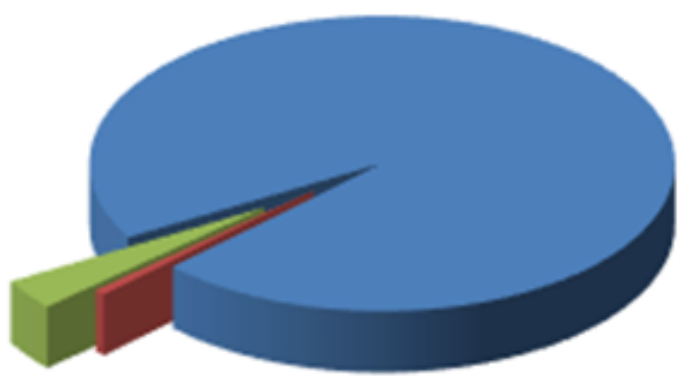

- Caries

Impactions

Dental anomalies

Figure 5 Frequency of dental problems in \%.

\section{Discussion}

Globally, the incidence of oral diseases differs depending upon the genetic makeup, social habits, socio economic status of country and the quality of health care provided at the lowest level. The aim of our study was to find out the norms in our population. Data of 1062 patients from the tertiary care centers including the National Institute of Child Health, with is the largest tertiary care facility for children in Karachi a city of 20 million from June 2012-December 2014 were included. Around 13 categories based on surgical sieve were made including trauma as the objective was to document the burden of oral diseases and factors risking the oral health. The performa was designed to include the age and gender of patients to observe diseases affecting specific age group and gender. The male to female ratio was 1.4: 1 with 617 males and 445 females included (Figure 3). Comparing it to the stats of Systemic analysis done by W. Marcenes in 2010 in an effort to find out the global burden of diseases, the ratio was 1.07 derived from 7805 males and 7265 females is bit higher. ${ }^{2}$ While it is almost similar to findings from study by Ogunlewe et al. ${ }^{18}$ with male to female ratio to be $1.5: 1{ }^{18}$ Though the findings of another study done in America were similar having higher percentage of males attending dental care centers but ratio is not quoted. ${ }^{20}$ The commonest category was the dental problems with dental caries contributing to 475 cases [44.7\%] followed by trauma [195 cases] and congenital/hereditary anomalies [162 cases] (Figure 5). It is similar to findings of studies done in Middle East, Baghdad, Brazil and the Systemic review done by Petersen $\mathrm{PE}^{2}$ and Marcenes $\mathrm{W}$ et al. ${ }^{7}$ making dental Caries the most common oral diseases found in all age groups. ${ }^{2-9}$ It is the leading cause of Dental Extractions in permanent dentition whiles the 3rd most common in deciduous dentition. ${ }^{1-17}$ Trauma is the second common reason for reporting with lacerations mostly. Trauma leading to hard tissue fracture shows trend towards more mandible fractures with highest number of patients with body of mandible fracture followed by multiple site fractures of mandible. Large number of patients between 
10-11 years of age diagnosed with fractures. All these findings are similar to the studies conducted in Nigeria and Baghdad. ${ }^{18,19}$ Though the main reason for patients reporting with traumatic injuries were road traffic accidents in age group 5-17 years while under 5 years of age fall injuries prevailed again similar to previous study done by Ali DA and differed from the findings of Bede SY. ${ }^{18,19}$ Hereditary or congenital problems were the third reason with $98 \%$ of the cases of Cleft Lip/Palate. Since NICH [National institute of child health] have cleft patients referred to their dental OPD not only from inside the hospital but also from other areas of Sindh, run by an orthodontics deals almost exclusively with cleft patients. Most of the cases reported at day 1 after birth thus, contributing to the high number of patients less than 1 year of age. The male to female ratio was 1:1.1, though most of the cases of cleft lip were common in females while male had cleft lip with palate prevalent. The frequency was found to be $15 \%$ of our sample which is very high in comparison to previously reported figures which were 3.75 per 1000 live births in Americans, 1:600 to 1:700 live births in Europeans, $0.82-4.04$ per 1000 live births in Asians, 0.9-2.69 per 1000 live births in Caucasians and 0.18-1.67 per live births in Africans. ${ }^{2}$ The ratio described in Asians is very high in comparison to others. Though it is low in comparison to a study conducted in northern Pakistan reporting 117 per 61,156 live births with Cleft lip making $42 \%$, Isolated cleft palate $24 \%$ and combined cleft lip and palate deformities $34 \%$ of the 117 cases. ${ }^{21}$ Also only 3 cases of other congenital problems including crouzon syndrome, fibrous dysplasia and encephalocele attended the Tertiary Care facility in comparison to 11 cases reported in the previous report. ${ }^{21}$ The fourth most reported oral disease was pre-cancerous lesion particularly the OSF contributing to $98.6 \%$ [ 68 cases] of the 69 cases with the youngest child of 7 years reporting with disease. This is mentioned especially here to remind the readers that, in this part of the subcontinent panchalia eating habits are common among the elderly with markedly increased trend of chalia, supari or gutka eating habits in children while in the rest of the world most common pre-cancerous lesion is Erythroplakia. ${ }^{2}$ Another interesting finding was the most number of patients reported were less than 1 year of age contributing to $15 \%$ followed by cases reported between $11-12$ years [8.1\%] and 9-10 years $(7.7 \%)$ (Figure 4$)$. The age group with lowest number of cases was between $13-14$ years of age with only $2.86 \%$ of cases studies. The highest number of cases reported differed across from 13.5 years to 12-15 years of age. ${ }^{18,20}$ The studies of Petersen ${ }^{2}$ and Marcenes $W$ et al. and Ali DA ${ }^{8}$ didn't have any findings depicting the number of cases according to age, thus comparison is not possible. ${ }^{2-8}$ The limitation of our study was the data collected was only from selected tertiary care facilities. To have a national profile we need to conduct a large multi-center regional study. To get a bigger a better picture a much larger sample will be required at each center to have a real impact. Also incomplete data comprising of more than 100 patients were not included which could have major impact on the facts and figures. It is the first step or the corner stone in building national pediatric oral disease burden database. This study is also the first documentation of pediatric oral diseases. Previous studies done in effort to find out the global burden of oral diseases included data from pediatric as well as adult population. ${ }^{2,7}$ As pediatric population contributes $30 \%$ of total population of the world and $43 \%$ of total population of Pakistan, a pediatric Oral disease profile is a necessity. ${ }^{22,23}$ To enhance the quality of care provided it is also important to develop the subspecialty Pediatric Oral Maxillofacial Surgery in Pakistan so that to produce skilled and knowledgeable surgeons that deals exclusively with children. Also an awareness program should be arranged so that prompt recognization of urgent care requiring problems should be correctly and promptly referred to the nearest care giving facility. Genetically and Habit related problems can be dealt with proper counseling and campaign.

\section{Acknowledgments}

None.

\section{Funding}

None.

\section{Conflicts of interest}

The authors declare there is no conflict of interests.

\section{References}

1. Richards D. Oral diseases affect some 3.9 billion people. Evid Based Dent. 2013;14(2):35.

2. Marcenes W, Kassebaum NJ, Bernabe E, et al. Global burden of oral conditions in 1990-2010: a systematic analysis. J Dent Res. 2013;92(7):592-597.

3. Jin LJ, Lamster IB, Greenspan JS, et al. Global burden of oral diseases: emerging concepts, management and interplay with systemic health. Oral Dis. 2016;22(7):609-619.

4. Igoumenakis D, Logothetis I, Barmpagadaki A, et al. Temporal space lymphatic malformation in a 15 -year-old adolescent: an extraordinary case. Journal of maxillofacial and oral surgery. 2016;15(Suppl 2):274278.

5. Rath R, Kaur S, Baig SA, et al. Multifocal head and neck neurofibromas with osseous abnormalities and muscular hypoplasia in a child with neurofibromatosis: type I. Case reports in radiology. 2016:1-7.

6. Khotani AA, Naimi AA, Albadawi E, et al. Prevalence of diagnosed temporomandibular disorders among Saudi Arabian children and adolescents. $J$ Headache Pain. 2016;17:41.

7. Petersen PE, Bourgeois D, Ogawa $\mathrm{H}$, et al. The global burden of oral diseases and risks to oral health. Bull World Health Organ. 2005;83(9):661-669.

8. Ali DA. Evaluation of Dental Status of Adolescents at Kuwait University Dental Clinic. Oral Health Prev Dent. 2016;14(2):183-188.

9. Moimaz SA, Borges HC, Saliba O, et al. Early Childhood Caries: Epidemiology, Severity and Sociobehavioural Determinants. Oral Health Prev Dent. 2016;14(1):77-83.

10. Petersen PE. Global policy for improvement of oral health in the 21st century-implications to oral health research of World Health Assembly 2007, World Health Organization. Community Dent Oral Epidemiol. 2009;37(1):1-8.

11. Petersen PE. World Health Organization global policy for improvement of oral health-World Health Assembly 2007. Int Dent J. 2008;58(3):115121.

12. Baelum V, Palenstein VHW, Hugoson A, et al. A global perspective on changes in the burden of caries and periodontitis: implications for dentistry. J Oral Rehabil. 2007;34(12):872-906.

13. Listl S, Galloway J, Mossey PA, et al. Global Economic Impact of Dental Diseases. Journal of dental research. 2015;94(10):1355-1361. 
14. Kassebaum NJ, Bernabe E, Dahiya M, et al. Global burden of untreated caries: a systematic review and metaregression. $J$ Dent Res. 2015;94(5):650-658.

15. Kassebaum NJ, Bernabe E, Dahiya M, et al. Global burden of severe periodontitis in 1990-2010: a systematic review and meta-regression. $J$ Dental Res. 2014;93(11):1045-1053.

16. Kassebaum NJ, Bernabe E, Dahiya M, et al. Global Burden of Severe Tooth Loss: A Systematic Review and Meta-analysis. J Dental Res. 2014;93(7 Suppl):20S-28S

17. Al Abdallah M, Al Hadidi A, Hammad M, et al. Prevalence and distribution of dental anomalies: A comparison between maxillary and mandibular tooth agenesis. A JO-DO. 2015;148(5):793-798.

18. Ogunlewe MO, James O, Ladeinde AL, et al. Pattern of paediatric maxillofacial fractures in Lagos, Nigeria. Int $J$ Paediatr Dent. 2006;16(5):358-362.
19. Bede SY, Ismael WK, Al Assaf D. Patterns of Pediatric Maxillofacial Injuries. Journal of Craniofacial surgery. 2016;27(3):e271-e275.

20. Al SAH, Lim CA, Singh YK, et al. Pediatric Maxillofacial Trauma: A Review of 156 Patients. Journal of oral and maxillofacial surgery: official journal of the American Association of Oral and Maxillofacial Surgeons. 2016;74(7):1420.e1-e4.

21. Elahi MM, Jackson IT, Elahi O, et al. Epidemiology of cleft lip and cleft palate in Pakistan. Plast Reconstr Surg. 2004;113(6):1548-1555.

22. Population census. 1950.

23. Global population at a glance:2002 and beyond. 2004:1-4 